\title{
Satisfacción con la vida de niños con trastorno por déficit de atención/hiperactividad: estudio de posibles factores de riesgo y de protección
}

\author{
Ana Miranda-Casas, M. Jesús Presentación-Herrero, Carla Colomer-Diago, Belén Roselló
}

Introducción. La psicología positiva ha potenciado el interés en el estudio de indicadores adaptativos, entre los que se incluye la satisfacción con la vida.

Objetivo. Analizar las relaciones de las percepciones de satisfacción con la vida de niños con trastorno por déficit de atención/hiperactividad (TDAH) y de sus padres con variables conductuales de riesgo y de protección, y determinar el poder predictivo de estas variables.

Pacientes y métodos. 56 padres y sus hijos con TDAH cumplimentaron cuestionarios de satisfacción con la vida, autoconcepto, coherencia, cualidades de adaptación y escalas de estimación conductual.

Resultados. Los problemas de conducta mantienen una relación negativa y significativa con la satisfacción con la vida; las relaciones de la satisfacción con la vida con el autoconcepto y con cualidades de adaptación son positivas. Los problemas sociales y el control se mantienen en los modelos de predicción para padres, mientras que el autoconcepto fue el factor con más poder predictivo en los modelos de predicción de satisfacción con la vida de los niños.

Conclusiones. Es importante incluir la identificación de factores relacionados con la satisfacción con la vida en la evaluación e intervención en personas con TDAH.

Palabras clave. Autoconcepto. Coherencia. Cualidades de adaptación. Problemas sociales. Satisfacción con la vida. TDAH.

\section{Introducción}

Se está produciendo en el momento presente una expansión exponencial de la psicología positiva, que constituye el contrapunto necesario al énfasis histórico que la psicología ha puesto en el estudio de las condiciones psicopatológicas. La nueva corriente ha enfatizado el uso de indicadores positivos de funcionamiento en la investigación como la calidad de vida (CAV), que incide no sólo en aspectos cuantificables (acceso a recursos médicos, oportunidades de ocio, nivel de renta, etc.), sino también en valoraciones internas como los juicios de satisfacción con la vida [1].

\section{Calidad de vida de los niños con trastorno por déficit de atención/hiperactividad}

La CAV consiste en la percepción subjetiva del estado de salud, incluyendo la enfermedad y el tratamiento, sobre el funcionamiento físico, psicológico y social [2]. El concepto de satisfacción con la vida se enmarca en el concepto más amplio de CAV. De hecho, es un proceso evaluativo cognitivo que se define como la evaluación global que hace la persona de su CAV de acuerdo con sus propios estándares [3].

En los últimos años ha aumentado la evidencia sobre el impacto negativo del trastorno por déficit de atención/hiperactividad (TDAH) en el bienestar de los niños y adolescentes y en su capacidad para el funcionamiento diario.

La reciente revisión realizada por Danckaerts et al [4] aporta una serie de conclusiones relevantes sobre el tema. Un primer punto que se debe destacar es que, salvo raras excepciones, los dominios de CAV son más negativamente valorados por niños y adolescentes con TDAH, en comparación con los niños sin problemas. Por otra parte, las valoraciones de los chicos con TDAH son más positivas que las de sus padres, por lo que debe obtenerse información, siempre que sea posible, tanto de los padres como de sus hijos para conseguir una visión más ajustada a la realidad.

Otra aportación importante del trabajo de Danckaerts et al [4] se refiere a la correlación negativa entre la CAV y la estimación de síntomas de hiperactividad/impulsividad e inatención por parte de padres y de profesores. Además, los niños con TDAH
Universitat Jaume I; Castellón (M.J. Presentación-Herrero). Universitat de València (A. Miranda-Casas, C. ColomerDiago). Servicio de Neuropediatría Hospital Universitario La Fe (B. Roselló); Valencia, España.

Correspondencia Dra. Ana Miranda Casas. Departamento de Psicología Evolutiva y de la Educación. Universitat de València. Avda. Blasco Ibáñez, 21. E-46010 Valencia. Fax: +34963983880

E-mail:

ana.miranda@uv.es

Financiación:

Plan Nacional I+D+I (FEDER y el Ministerio de Ciencia y Tecnología) ref. EDU 2009-07672.

Agradecimientos:

A las familias que dedicaron parte de su tiempo a aportar la información necesaria para este trabajo.

Aceptado tras revisión externa: 20.01.11.

Cómo citar este artículo: Miranda-Casas A, PresentaciónHerrero MJ, Colomer-Diago C, Roselló B. Satisfacción con la vida de niños con trastorno por déficit de atención/hiperactividad: estudio de posibles factores de riesgo y de protección. Rev Neurol 2011; 52 (Supl 1): S119-26.

(c) 2011 Revista de Neurología 
más un trastorno comórbido (especialmente oposicionismo desafiante y problemas de conducta) presentan peor CAV que los que tienen sólo TDAH. Los resultados sobre dificultades del aprendizaje (DA) muestran más contradicciones. Algunas investigaciones [5] han encontrado en niños con DA y TDAH un panorama más disfuncional en los siguientes dominios: impacto emocional sobre los padres, impacto en la disponibilidad de tiempo de los padres, comportamiento, autoestima y funcionamiento psicosocial general (edad de los participantes entre 11 y 12 años). Por el contrario, en otros trabajos [6] la comorbilidad con DA no tuvo una incidencia negativa sobre la valoración de la CAV de niños con TDAH. La variable edad puede actuar como un factor modulador de los resultados, ya que los padres de niños más pequeños (6-8 años) aún no tienen tanta conciencia de la trascendencia de las DA en la posición sociolaboral futura del niño como los padres de chicos más mayores.

En cuanto a la comparación de la CAV de niños con TDAH con la de niños que sufren otras enfermedades, la conclusión general es que el TDAH tiene un impacto total sobre la CAV similar al de otras enfermedades físicas como el cáncer, el asma o la parálisis cerebral. No obstante, el TDAH tiene un impacto mayor en el área psicosocial y menor en el área física que las enfermedades físicas crónicas.

La influencia de factores demográficos y de historia familiar en la CAV de niños con TDAH también se puso de manifiesto en la revisión de Danckaerts et al [4]. Así, tener un padre con problemas mentales y no vivir con ambos padres están asociados con una CAV más pobre; no obstante, en general los resultados no señalan que factores como número de hijos que viven en el hogar, historia parental de TDAH, consumo de drogas/alcohol durante el embarazo y bajo peso o prematuridad influyan claramente en la CAV de niños con TDAH.

\section{Satisfacción con la vida de niños con trastorno por déficit de atención/hiperactividad: factores de protección}

La CAV tiene un radio de acción más amplio que el concepto de satisfacción con la vida y atiende menos a la dimensión del bienestar subjetivo, de ahí que la investigación sobre este tópico haya analizado sobre todo las variables que pueden influir de forma negativa en la CAV; sin embargo, los pocos trabajos empíricos que han intentado profundizar en las percepciones de satisfacción con la vida de personas con TDAH han procurado dilucidar las cualidades que intervienen para lograr resultados de éxito.
Luthar y Zelazo [7] definen la resiliencia como un fenómeno que refleja la adaptación positiva del niño a pesar de estar inmerso en situaciones de riesgo. Los factores de resiliencia son esenciales para lograr la satisfacción vital. Los resultados de un excelente trabajo del Centro Frostig [8] en el que participaron estudiantes con DA permitieron identificar un conjunto de atributos que diferenciaban significativamente a los sujetos con una adaptación exitosa a la vida adulta de los que no lo lograron: autoconciencia, control, perseverancia, planteamiento de metas, disposición a utilizar apoyos sociales y estrategias de control emocional.

Otro indicador de resiliencia y fortaleza personal es el sentido de coherencia (SEC), la forma de considerarse a sí mismo en el mundo. El SEC se ha estudiado en grupos que sufren diversos tipos de estresores $y$, en particular, enfermedades físicas como la parálisis cerebral o la fibromialgia, mientras que las investigaciones con niños con TDAH son raras. Destacan en este sentido dos aportaciones. Un trabajo [9] realizado con universitarios con distintos problemas -entre los que destacaba por su incidencia (46\% de la muestra) el TDAH y/o las DA- encontró una correlación positiva entre el SEC y las valoraciones de satisfacción con la vida. El segundo trabajo -desarrollado por investigadores del Instituto Karolinska [10]- concluyó que el SEC elevado constituía un factor protector de la evolución del TDAH a largo plazo. Las puntuaciones más altas de TDAH a los 16 años estaban asociadas a los 21 años con una gravedad mayor de las puntuaciones de TDAH. Esta relación era más fuerte cuando el SEC era más bajo. Por el contrario, un SEC mejor (esto es, más alto a los 16 años) estaba relacionado con puntuaciones más bajas en manifestaciones de TDAH a los 21 años.

Resumiendo, la revisión de la bibliografía sugiere que no existe un único factor que pueda explicar cuán satisfactoria perciben su vida los niños con TDAH. Más bien parece producirse un efecto acumulativo de múltiples variables, de riesgo y de protección, que interactúan y dan lugar al resultado final.

\section{Objetivo}

Esta investigación no pretende realizar un análisis exhaustivo del conjunto de correlatos y predictores de la satisfacción con la vida de niños y adolescentes con TDAH. Con un enfoque microanalítico, pragmático y fundamentalmente exploratorio, los objetivos del presente trabajo, que se circunscriben en el dominio de factores psicológicos 
individuales de riesgo y de protección, son los siguientes:

- Determinar la concordancia que existe entre las valoraciones de la satisfacción con la vida de los padres y de los niños con TDAH.

- Analizar las relaciones de las percepciones de satisfacción con la vida valorada por los niños y por los padres con variables conductuales de riesgo y de protección.

- Explorar cuáles de estos factores tienen un poder significativo para predecir la satisfacción con la vida de los niños y de los padres.

\section{Pacientes y métodos}

\section{Muestra}

En el presente estudio participaron 56 familias con un niño con un diagnóstico de TDAH, subtipo combinado. De la muestra total, 54 eran varones y 2 mujeres de entre 7 y 16 años (media: 11,14 $\pm 2,23$ ), con un cociente intelectual mayor de 70 (media: $104,54 \pm 16,65)$. En la mayoría de los casos (65\%) se trataba de pacientes del Servicio de Neuropediatría del Hospital La Fe de Valencia. Se adoptaron los siguientes criterios para confirmar el diagnóstico: valoración de padres y de profesores de seis o más de los síntomas de inatención y de hiperactividad/impulsividad del Manual diagnóstico y estadístico de los trastornos mentales, $4 .^{a}$ edición, texto revisado (DSM-IV-TR) [11], duración de los síntomas superior a un año y manifestaciones del problema antes de los 7 años. Los criterios de exclusión aplicados fueron: $\mathrm{CI}<70$-evaluado mediante la escala de inteligencia para niños de Wechsler revisada (WISC-R) [12]-, un diagnóstico de esquizofrenia o trastorno generalizado del desarrollo, daño neurológico, sensorial o déficit motores.

Utilizando como criterio una puntuación de $T>63$ en la escala de Conners revisada para padres (CPRS-R) [13], un 43\% de los niños tenía problemas de oposicionismo; un $37 \%$, ansiedad; un $43 \%$, problemas sociales, y un $46 \%$, alta labilidad emocional. Los padres en su mayoría eran profesionales (un 12,9\%, padres; un 16,1\%, madres), desempeñaban funciones administrativas (un 27,4\%, padres; un $12,9 \%$, madres) o trabajaban en el área de servicios (un $16,1 \%$, padres; un $25,8 \%$, madres).

\section{Medidas y procedimiento}

\section{Satisfacción con la vida}

- Escala breve multidimensional de satisfacción con la vida para niños (BMSLSS [14]). Este instrumento se fundamenta en un modelo jerárquico de satisfacción con la vida en el cual cinco dominios específicos de segundo orden (familia/amigos/experiencia escolar/personal y entorno físico) se incluyen bajo un factor general de satisfacción con la vida. Las alternativas de respuesta a los ítems oscilan entre 1 (horrible) y 7 (maravillosa); por ejemplo, 'yo describiría mi vida familiar como.... La escala ha demostrado tener una consistencia interna de 0,85 y mantener altas correlaciones con medidas de satisfacción de vida global y variables relacionadas.

- Escala breve multidimensional de satisfacción con la vida para padres. Se trata de la adaptación de la BMSLSS [14] para su cumplimentación por los padres. El objetivo es recabar información sobre las percepciones que tienen los padres de la satisfacción con la vida de sus hijos; por ejemplo, 'considera que su hijo valora la escuela y los estudios como...; 'piensa que su hijo valora la vida familiar como...'

\section{Variables conductuales}

- CPRS-R [13]. Esta escala está validada para niños y adolescentes de entre 3 y 17 años de edad y consta de 80 ítems medidos en una escala tipo Likert, con puntuaciones entre 0 (nunca) y 3 (muy a menudo), que están agrupados en 14 subescalas: oposicionismo; problemas cognitivos/inatención; hiperactividad/impulsividad; timidez/ansiedad; perfeccionismo; problemas sociales; problemas psicosomáticos; índice de TDAH; índice de inquietud/impulsividad; índice de labilidad emocional; índice global de problematicidad; DSM-IV, falta de atención; DSM-IV, hiperactividad/impulsividad y DSM-IV, total. Las tres últimas escalas referidas al DSM-IV no se utilizaron en los análisis. Las escalas de Conners se encuentran entre los instrumentos de medida para el TDAH con mejor estandarización de muestras, evidencia de validez y fiabilidad [15].

\section{Variables de protección}

- Cualidades de adaptación (adaptado de [16]). Este instrumento, que fue cumplimentado por los padres, consta de seis subescalas que evalúan diferentes habilidades:

a) Autoconciencia: reconocimiento realista de las capacidades y limitaciones (por ejemplo, 'cuando tiene que estudiar o hacer trabajos sabe cuáles son sus dificultades más importantes').

b) Control: creencia de control sobre el propio 
Tabla I. Correlaciones entre las variables de satisfacción con la vida percibida por el niño, satisfacción con la vida percibida por los padres y las variables conductuales de riesgo.

\begin{tabular}{|c|c|c|c|c|c|c|c|c|c|c|c|}
\hline & 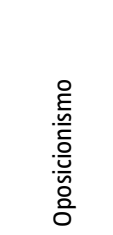 & 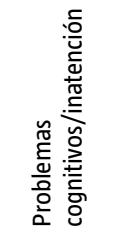 & 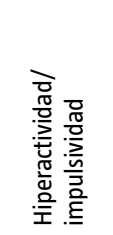 & 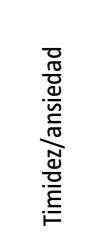 & 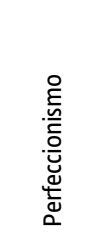 & 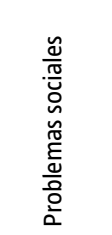 & 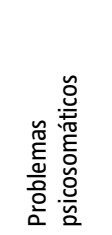 & 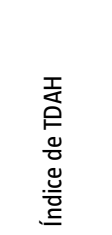 & 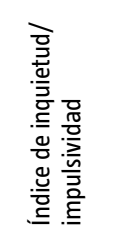 & 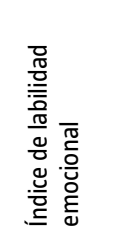 & 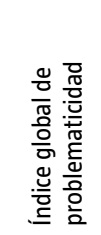 \\
\hline Niño & $-0,564^{b}$ & $-0,355^{b}$ & $-0,365^{b}$ & $-0,245$ & $-0,199$ & $-0,202$ & $-0,253$ & $-0,362^{b}$ & $-0,373^{b}$ & $-0,346^{b}$ & $-0,452^{b}$ \\
\hline dres & $-0,485^{b}$ & $-0,312^{\mathrm{a}}$ & $-0,383^{b}$ & $-0,196$ & 0,040 & $-0,404^{b}$ & $-0,466^{b}$ & $-0,205$ & $-0,287^{a}$ & $-0,255$ & $-0,405^{b}$ \\
\hline
\end{tabular}

${ }^{a} p<0,05 ;{ }^{b} p<0,01$ TDAH: trastorno por déficit de atención/hiperactividad.

destino (por ejemplo, 'sabe que conseguir lo que quiere depende mucho de él').

c) Perseverancia: persistencia para lograr metas a pesar de las dificultades y reconocimiento del valor de la adversidad (por ejemplo, 'aunque le cueste, cuando quiere conseguir algo se empeña y no abandona').

d) Planteamiento de metas: habilidad para establecer metas realistas y estrategias necesarias para alcanzarlas (por ejemplo, 'las cosas que se propone conseguir suelen estar a su alcance').

e) Disposición para usar apoyos (por ejemplo, 'sabe distinguir cuándo debe esforzarse por sí mismo y cuándo tiene que pedir ayuda').

f) Estrategias de afrontamiento: desarrollo de medios para reducir y afrontar el estrés y la frustración, manteniendo una actitud positiva (por ejemplo, 'aunque sea difícil, sabe que puede aprender a tranquilizarse').

- Escala de sentido de coherencia para niños [17]. Incluye 16 ítems que describen los sentimientos de confianza que tiene el niño en su mundo, expresada por la sensación de comprensión de su ambiente (por ejemplo, 'siento que no sé qué hacer en clase'), sentimientos de confianza en que cuando necesite ayuda la tendrá (por ejemplo, 'cuando yo quiero algo estoy seguro de que lo conseguiré') y por su sensación de motivación en dedicar esfuerzos a la realización de diferentes tareas (por ejemplo, 'yo estoy interesado en muchas cosas'). Los ítems se valoran mediante una escala de cuatro puntos que oscila entre nunca (1) y siempre (4). La escala ha obtenido un $\alpha$ de Cronbach de 0,75.

- Escala de autoconcepto (SDQ-I), adaptación de [18]. Del instrumento multidimensional que mide ocho dimensiones del autoconcepto y cuenta con indicadores psicométricos de fiabilidad y validez, se seleccionó para este estudio la escala de autoconcepto general. El cuestionario está integrado por 10 frases descriptivas que expresan características personales globales a las que el niño debe responder según una escala de 5 puntos entre $1=$ falso y 5 = verdad (por ejemplo, 'en general me gusta ser como soy', 'tengo muchas cualidades buenas', etc.).

La evaluación se realizó con el consentimiento informado de las familias. Los pacientes que tomaban medicación $(67,9 \%)$ dejaron de tomarla 48 horas antes de la evaluación.

\section{Análisis}

Todos los análisis estadísticos se llevaron a cabo con el programa SPSS v. 17.0. En primer lugar, se realizaron correlaciones de Pearson entre la satisfacción con la vida percibida por los niños y la percibida por los padres. A continuación se calcularon las correlaciones entre todas las variables en estudio y después se llevaron a cabo análisis de regresión jerárquica por pasos sucesivos para determinar el poder de las variables conductuales y cognitivoafectivas para predecir la satisfacción percibida por el niño y por los padres. Las variables independientes se introdujeron en la ecuación de regresión en dos pasos sucesivos; primero las variables conductuales de riesgo (oposicionismo, problemas cognitivos/inatención, hiperactividad/impulsividad, problemas sociales, problemas psicosomáticos, índice de TDAH, índice de inquietud/impulsividad, índice de labilidad emocional e índice global de problematicidad) y después los factores de protección (auto- 
conciencia, control, perseverancia, metas, apoyo, estrategias de afrontamiento, coherencia y autoconcepto). Posteriormente se invirtió el orden de introducción de las variables: primero los factores adaptativos y después las variables conductuales a fin de comprobar qué modelo tenía un poder explicativo superior.

\section{Resultados}

La correlación entre las percepciones de satisfacción con la vida de los niños y de los padres es positiva y significativa $(r=0,534 ; p=0,000)$, con un valor moderado. Además, la media de las percepciones de los niños $(28,38)$ es significativamente superior a la media $(22,34)$ de las percepciones de los padres $(t=9,559 ; p=0,000)$

Como puede comprobarse en la tabla I, la satisfacción con la vida de los niños mantiene correlaciones negativas significativas con oposicionismo $(r=-0,564 ; p=0,000)$, problemas cognitivos/inatención $(r=-0,355 ; p=0,007)$, hiperactividad/impulsividad ( $r=-0,365 ; p=0,006)$, índice de TDAH $(r=-0,362 ; p=0,006)$, índice de inquietud/impulsividad $(r=-0,373 ; p=0,005)$, índice de labilidad emocional $(r=-0,346 ; p=0,009)$ y con el índice global de problematicidad ( $r=-0,452 ; p=0,000)$. Por otra parte, la satisfacción percibida por los padres correlaciona negativa y significativamente con oposicionismo $(r=-0,485 ; p=0,000)$, problemas cognitivos/inatención $(r=-0,312 ; p=0,019)$, hiperactividad/impulsividad $(r=-0,383 ; p=0,009)$, problemas sociales $(r=-0,404 ; p=0,002)$, psicosomáticos $(r=-0,466 ; p=0,000)$, índice de inquietud/ impulsividad $(r=-0,287 ; p=0,032)$ e índice global de problematicidad $(r=-0,405 ; p=0,002)$.

En la tabla II se presentan las correlaciones entre la satisfacción con la vida percibida por los niños y por los padres y las variables de protección. La satisfacción de los niños correlaciona positiva y significativamente con autoconcepto $(r=0,730 ; p=$ $0,000)$, autoconciencia $(r=0,318 ; p=0,017)$, control $(r=0,357 ; p=0,007)$, perseverancia $(r=0,334$; $p=0,012)$, apoyo $(r=0,292 ; p=0,029)$ y estrategias de afrontamiento $(r=0,270 ; p=0,044)$; pero no con metas $(r=0,193 ; p=0,155)$ ni coherencia $(r=$ $-0,050 ; p=0,715)$; mientras que la satisfacción de los padres muestra correlaciones positivas significativas con todas las variables de protección -autoconciencia $(r=0,425 ; p=0,001)$, control $(r=0,522$; $p=0,000)$, perseverancia $(r=0,521 ; p=0,000)$, me$\operatorname{tas}(r=0,507 ; p=0,000)$, apoyo $(r=0,564 ; p=0,000)$, estrategias de afrontamiento $(r=0,555 ; p=0,000) \mathrm{y}$
Tabla II. Correlaciones entre las variables de satisfacción con la vida percibida por el niño y satisfacción con la vida percibida por los padres con las variables de protección.

\begin{tabular}{|c|c|c|c|c|c|c|c|c|}
\hline & 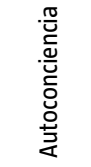 & $\begin{array}{l}\overline{0} \\
\text { 녿 } \\
\text { O }\end{array}$ & 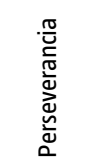 & 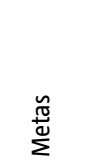 & $\begin{array}{l}\text { Oे } \\
\text { 운 }\end{array}$ & 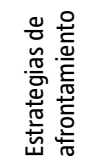 & 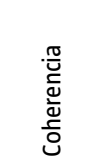 & 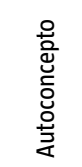 \\
\hline Niño & $0,318^{a}$ & $0,357^{b}$ & $0,334^{a}$ & 0,193 & $0,292^{a}$ & $0,270^{a}$ & $-0,050$ & $0,730^{b}$ \\
\hline Padres & $0,425^{b}$ & $0,522^{b}$ & $0,521^{b}$ & $0,507^{b}$ & $0,564^{b}$ & $0,555^{b}$ & $-0,034$ & $0,311^{a}$ \\
\hline
\end{tabular}

${ }^{\mathrm{a}} p<0,05 ;{ }^{\mathrm{b}} p<0,01$.

autoconcepto $(r=0,311 ; p=0,020)$ - exceptuando la coherencia $(r=-0,034 ; p=0,804)$.

Por último, la tabla III muestra los modelos predictivos para la satisfacción con la vida percibida por los niños y la percibida por los padres.

Los resultados del análisis de regresión muestran que, cuando se introduce en primer lugar el bloque de variables conductuales, la satisfacción total de los niños está predicha por tres variables que explican un $68,6 \%$ de la varianza: oposicionismo $\left(\Delta \mathrm{R}^{2}=0,254\right.$; $p=0,000)$, autoconcepto $\left(\Delta \mathrm{R}^{2}=0,386 ; p=0,000\right) \mathrm{y}$ autoconciencia $\left(\Delta R^{2}=0,046 ; p=0,000\right)$. Cuando en el análisis de regresión se introdujo en primer lugar el bloque de variables de protección, el porcentaje total de varianza explicada fue del $70,6 \%$, e intervinieron tres variables: autoconcepto $\left(\Delta R^{2}=0,612\right.$; $p=0,000)$, estrategias de afrontamiento $\left(\Delta \mathrm{R}^{2}=0,060\right.$; $p=0,000)$ y coherencia $\left(\Delta \mathrm{R}^{2}=0,034 ; p=0,000\right)$.

Para la satisfacción percibida por los padres, el modelo de predicción al introducir primero las variables conductuales está formado por problemas sociales $\left(\Delta \mathrm{R}^{2}=0,287 ; p=0,000\right)$, índice global de problematicidad $\left(\Delta \mathrm{R}^{2}=0,112 ; p=0,000\right)$ y problemas psicosomáticos $\left(\Delta \mathrm{R}^{2}=0,055 ; p=0,000\right)$ y las variables de protección de apoyo $\left(\Delta \mathrm{R}^{2}=0,106 ; p=\right.$ $0,000)$ y control $\left(\Delta R^{2}=0,045 ; p=0,000\right)$. En conjunto el modelo explicaba un $60,5 \%$ de la varianza. $\mathrm{Al}$ introducir primero las variables de protección el modelo explicó un 55,7\% de la varianza, incluyendo tres variables: estrategias de afrontamiento $\left(\Delta \mathrm{R}^{2}=\right.$ $0,283 ; p=0,000)$, control $\left(\Delta \mathrm{R}^{2}=0,131 ; p=0,000\right) \mathrm{y}$ problemas sociales $\left(\Delta \mathrm{R}^{2}=0,143 ; p=0,000\right)$.

\section{Discusión}

Un objetivo de este trabajo fue analizar la concordancia entre las valoraciones de la satisfacción con 
Tabla III. Análisis de regresión jerárquica por pasos sucesivos de las variables conductuales de riesgo y de protección con la satisfacción con la vida percibida por el niño y con la satisfacción de vida percibida por los padres.

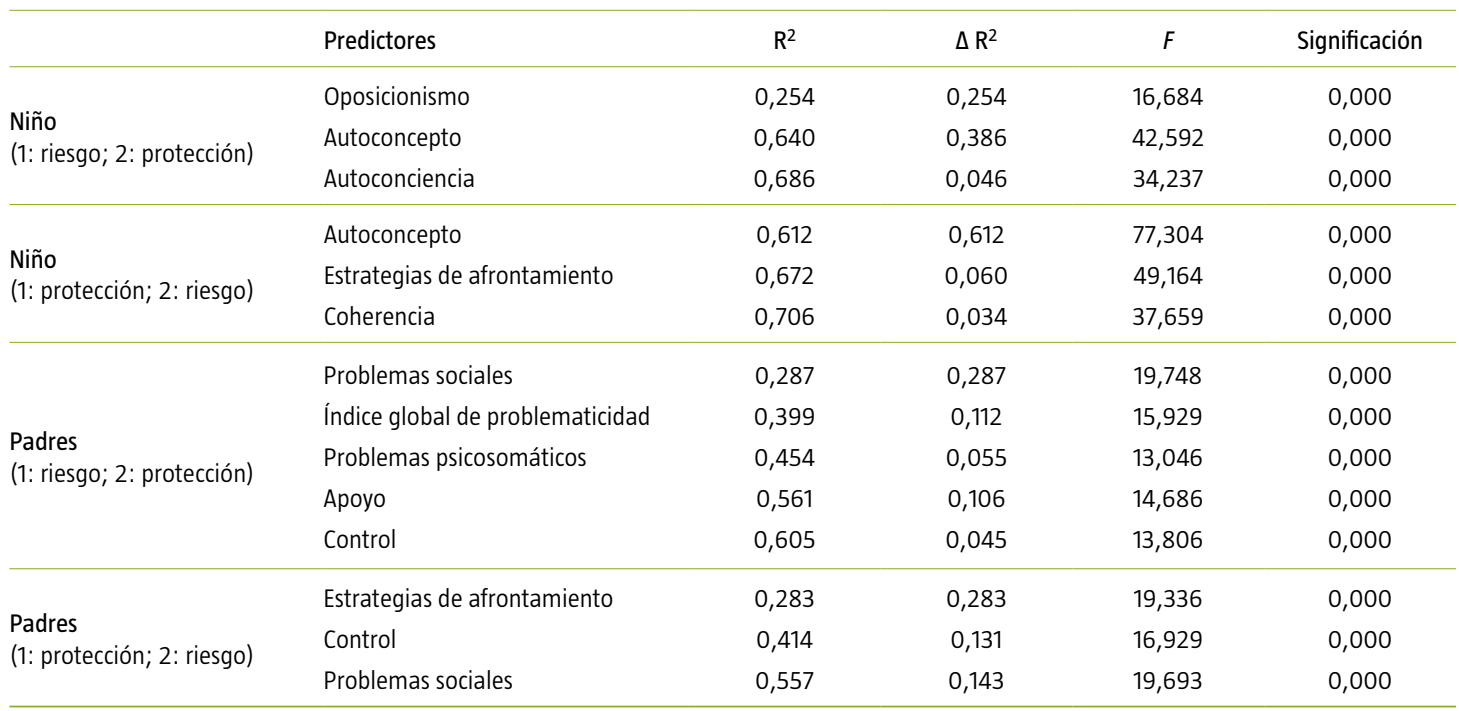

la vida de los padres y las de los niños con TDAH. Las correlaciones presentaron valores moderados. Un punto que se debe destacar es que la satisfacción con la vida valorada por los niños es significativamente más elevada que la estimada por sus padres, lo cual puede deberse a que la evaluación de los niños esté afectada por sesgos positivos ilusorios. Para conservar una autoimagen positiva los niños con TDAH suelen sobreestimar sus capacidades y su autoconcepto [19] y, de igual modo, pueden utilizar un mecanismo de protección, con lo que inflan la valoración de la felicidad que sienten con su vida. Por consiguiente, la estrategia más oportuna consistirá en utilizar las perspectivas de los padres y las de los niños para conseguir una visión más integrada.

Los resultados del segundo objetivo indican una mayor coherencia entre los dos informantes. Tanto en el caso de los padres como en el de los niños, la satisfacción con la vida está relacionada positiva y significativamente con todos los factores de protección, hallazgos que se suman a los de otros investigadores $[9,10]$. Por el contrario, la satisfacción con la vida percibida por los padres y la percibida por los niños, como parece lógico, mantiene correlaciones significativas y negativas con las conductas psicopatológicas evaluadas, tanto exteriorizadas como interiorizadas, a excepción de la ansiedad y el perfeccionismo. En la misma línea de la revisión de Danckaerts et al [4] sobre CAV, se obtienen correlaciones negativas entre síntomas de hiperactividad/ impulsividad e inatención y oposicionismo y percepciones de satisfacción con la vida de los padres y de los niños. La gravedad de las manifestaciones típicas del TDAH cuando se asocian al trastorno oposicionista desafiante o a problemas de conducta suponen un mayor riesgo de sufrir el rechazo de los compañeros, bajo rendimiento académico y baja autoestima [20,21]. Por tanto, resulta lógico que la satisfacción con la vida esté negativa y significativamente correlacionada con estas conductas.

El tercer objetivo fue identificar los factores que mejor predijeran la satisfacción con la vida evaluada por los niños y por los padres. La primera conclusión es que en los modelos de predicción sobre satisfacción con la vida estimada por los padres los problemas sociales y la capacidad de control que valoran en sus hijos se mantienen como variables predictivas importantes. Aparecen también otras dimensiones con sentido negativo (concretamente la problematicidad global y las quejas psicosomáticas) e indicadores de protección (como la utilización de apoyo y las estrategias de afrontamiento). Por otra parte, el autoconcepto es un componente que se mantiene en los dos modelos de predicción de satisfacción de los niños con la vida con un peso predictivo notable. Completan el panorama la autoconciencia, las estrategias de afrontamiento y el SEC junto con el oposicionismo. Por último, el desarrollo de medios para reducir y afrontar el estrés y la frustración, manteniendo una actitud positiva, es el aspecto que comparte la predicción de la satis- 
facción con la vida tanto de los padres como de sus hijos con TDAH.

Haber restringido el estudio de la satisfacción con la vida a factores psicológicos individuales es una importante limitación de esta investigación. Otro de los problemas que afectan a ésta es que sus resultados son básicamente correlacionales. Sería necesario confirmar los hallazgos llevando a cabo un estudio longitudinal que siguiera el curso evolutivo y las características de niños con TDAH que tienen una vida satisfactoria. A medida que estos factores se identifiquen y se comprendan con más precisión, podrán desarrollarse programas que tengan muchas probabilidades de éxito.

No obstante, a pesar de sus limitaciones, este estudio exploratorio aporta algunos conocimientos sobre dónde poner el acento para ayudar a los estudiantes con TDAH a lograr una vida más satisfactoria, productiva y gratificante. Los resultados sugieren que la intervención debe contemplar el desarrollo de los atributos personales adaptativos, ya que la disponibilidad de más recursos se asociará con resultados más positivos. El reconocimiento realista de las capacidades, limitaciones e intereses, el control emocional y el sentimiento de control personal aumentarán la probabilidad de superar con éxito las situaciones difíciles. También deberá promoverse la utilización de los apoyos sociales. Los vínculos con el profesor o con un mentor desarrollan la confianza mutua y generan sentimientos de que uno es comprendido, respetado y amado.

Otra meta será aumentar el autoconcepto mediante counseling: técnicas de relajación, entrenamiento en estrategias de afrontamiento, habilidades de comunicación y biblioterapia. También hay otros procedimientos que pueden implementarse en la clase, como la tutoría de compañeros, el aprendizaje cooperativo o, en un contexto más lúdico, el baile, el fitness y los deportes [22].

\section{Bibliografía}

1. Huebner ES, Suldo SM, Smith LC, McKnight C. Life satisfaction in children and youth: empirical foundations and implications for school psychologists. Psychol Sch 2004; 41:81-93.

2. Leidy NK, Revicki DA, Geneste B. Recommendations for evaluating the validity of quality of life claims for labeling and promotion. Value Health 1999; 2: 113-27.

3. Shin DC, Johnson DM. Avowed happiness as an overall assessment of the quality of life. Soc Indic Res 1978; 5: 475-92.
4. Danckaerts M, Sonuga-Barke EJS, Banaschewski T, Buitelaar J, Döpfner M, Hollis C, et al. The quality of life of children with attention deficit/hyperactivity disorder: a systematic review. Eur Child Adolesc Psychiatry 2010; 19: 83-105.

5. Karande S, Bhosrekar K. Impact of attention deficit/hyperactivity disorder on health-related quality of life of specific learning disability children. Indian J Pediatr 2009; 76: 1119-24.

6. Klassen AF, Miller A, Fine S. Health-related quality of life in children and adolescents who have a diagnosis of attentiondeficit/hyperactivity disorder. Pediatrics 2004; 114: 541-7.

7. Luthar SS, Zelazo LB. Research on resilience: an integrative view. In Luthar SS, ed. Resilience and vulnerability; adaptation in the context of childhood adversities. Cambridge: Cambridge University Press; 2003. p. 511-49.

8. Goldberg RJ, Higgins EL, Raskind MH, Herman KL. Predictors of success in individuals with learning disabilities: a qualitative analysis of a 20-year longitudinal study. Learn Disab Res Pract 2003; 18: 222-36.

9. Lustig DC, Rosenthal DA, Strauser DR, Haynes K. The relationship between sense of coherence and adjustment in persons with disabilities. Rehabil Couns Bull 2000; 43: 134-41.

10. Edbom T, Malmberg K, Lichenstein P, Granlund M, Larsson JO. High sense of coherence in adolescence is a protective factor in the longitudinal development of ADHD symptoms. Scand J Caring Sci 2010; 24: 541-7.

11. American Psychiatric Association. Manual diagnóstico y estadístico de los trastornos mentales, texto revisado. 4 ed. Barcelona: Masson; 2003.

12. Wechsler D. Escala de inteligencia para niños de Wechsler revisada (WISC-R). Madrid: TEA Ediciones; 1980.

13. Conners CK. Conners Parent Rating Scale-Revised: long version (CPRS-R:L). Canada: MHS; 2001.

14. Seligson JL, Huebner ES, Valois RF. Preliminary validation of the Brief Multidimensional Students' Life Satisfaction Scale (BMSLSS). Soc Indic Res 2003; 61: 121-45.

15. Demaray MK, Elting J, Schaefer K. Assessment of attentiondeficit hyperactivity disorder (ADHD). A comparative evaluation of five, commonly used, published rating scales. Psychol Sch 2003; 40: 341-61.

16. Raskind MH, Goldberg RJ, Higgins EL, Herman K L. Patterns of change and predictors of success in individuals with learning disabilities: results from a twenty-year longitudinal study. Learn Disabil Res Pract 1999; 14: 35-49.

17. Margalit M, Efrati M. Sense of coherence, companionship, and loneliness among children with learning disorders. In Biennial Meeting of the Society for Research on Child Development. Indianapolis, 30 de marzo a 2 de abril de 1995.

18. Núñez JC, González-Pumariega S, González-Pienda JA. Autoconcepto en niños con y sin dificultades de aprendizaje. Psicothema 1995; 7: 587-604.

19. Hoza B, Pelham WE, Dobbs J, Owens JS, Pillow DR. Do boys with attention-deficit/hyperactivity disorder have positive illusory self-concepts? J Abnorm Psyhol 2001; 111: 268-78.

20. Bussing R, Mason DM, Bell L, Porter P, Garvan C. Adolescent outcomes of childhood attention-deficit/hyperactivity disorder in a diverse community sample. J Am Acad Child Adolesc Psychiatry 2010; 49: 595-605.

21. Mannuzza S, Klein RG, Moulton JL. Lifetime criminality among boys with attention deficit hyperactivity disorder: a prospective follow-up study into adulthood using official arrest records. Psychiatry Res 2008; 160: 237-46.

22. Elbaum B, Vaughn S. School-based interventions to enhance the self-concept of students with learning disabilities: a meta-analysis. Elementary School Journal 2001; 101: 303-29. 
Satisfaction with life of children with attention deficit hyperactivity disorder: a study of possible protection and risk factors

Introduction. Positive psychology has boosted interest about the study of factors of adjustment, among which is included satisfaction with life.

Aim. To analyze the relationship between satisfaction with life of children with attention deficit hyperactivity disorder (ADHD) and their parents with behavioral risk and protective variables, and to determine the predictive power of these variables.

Patients and methods. 56 parents and their children with ADHD filled out questionnaires of satisfaction with life, selfconcept, sense of coherence, success attributes and behavioral rating scales.

Results. Behavioral problems have a significant negative relationship with satisfaction with life perceived by parents and by children while the relationships with the self-concept and success attributes have a positive value. The most predictive factors were social problems, oppositional behavior, self-concept, self-awareness and social support.

Conclusions. The identification of factors that put at risk or enhance satisfaction with life of people with ADHD will improve the functionality of evaluation and intervention processes.

Key words. ADHD. Coherence. Satisfaction with life. Self-concept. Social problems. Success attributes. 